\title{
Solitons bifurcations in presence of surface tension
}

\author{
André Fonseca*† \\ Universidade Federal do ABC, Centro de Matemática, Computação e Cognição, Rua Catequese \\ 242, 09090-400, Santo André, SP, Brazil, 5511 4992-1333 \\ E-mail: andre.fonseca@ufabc.edu.br
}

\begin{abstract}
We study the existence of solitons related to homoclinic solutions for reversible Hamiltonian systems, taking the family of differential equations $u^{i v}+a u^{\prime \prime}-u+f(u, b)=0$ as a model, where $f$ is an analytic function and $a, b$ real parameters. Applying a non pertubative approach which is a modification of the standard procedure of tracking intersections of unstable manifolds with some invariant set, we determine soliton solutions and also their bifurcations in the space of parameters giving a picture of the chaotic structural distribution to phase and amplitude shifts phenomena. In Section 1 we introduce a brief history of solitons, begining with the translation wave and its discoverer, John Scott Russell in 1834. In Section 2 we present our model equation, establish non restrictive hypotheses and state the main result based on reversibility properties, providing the algorithm results for solitons parameters and bifurcations. In Section 3 we discuss the model equation energy and geometry, pointing out the zero energy surface and Poincaré sections related to the main result and present in Section 4 our conclusion.
\end{abstract}

Fifth International Conference on Mathematical Methods in Physics - IC2006

April 24-28 2006

Centro Brasilerio de Pesquisas Fisicas, Rio de Janeiro, Brazil

\footnotetext{
* Speaker.

${ }^{\dagger}$ Thanks to Dr. Gerson Francisco from Theoretical Physics Institute of São Paulo State University IFT-UNESP, Brazil
} 


\section{Introduction}

Our work is about solitons or solitary waves, which are non-linear waves that travel without change of form or speed.

We can talk about solitons in different contexts: water wave problems [1], high temperature superconductors [2], energy transport in DNA [3], fibre-optic communications [4], and so on. But our concern is about solitons bifurcations in presence of surface tension, that is, water wave problems.

In figure 1 (a) one can see solitons at the Gibraltar Strait, when the Atlantic water is accelerated by its narrow passage. Then, the soliton packet travel miles without distortion through Mediterranean water while the boundary conditions remain the same. They were first seen from space on the Apollo-Soyuz mission in 1975. In the same figure (b) one can also find a beautiful picture which illustrates what happens when solitons travel in deeper water. In this this region they slow down, acummulationing theirselves, forming a Gauss distribution.

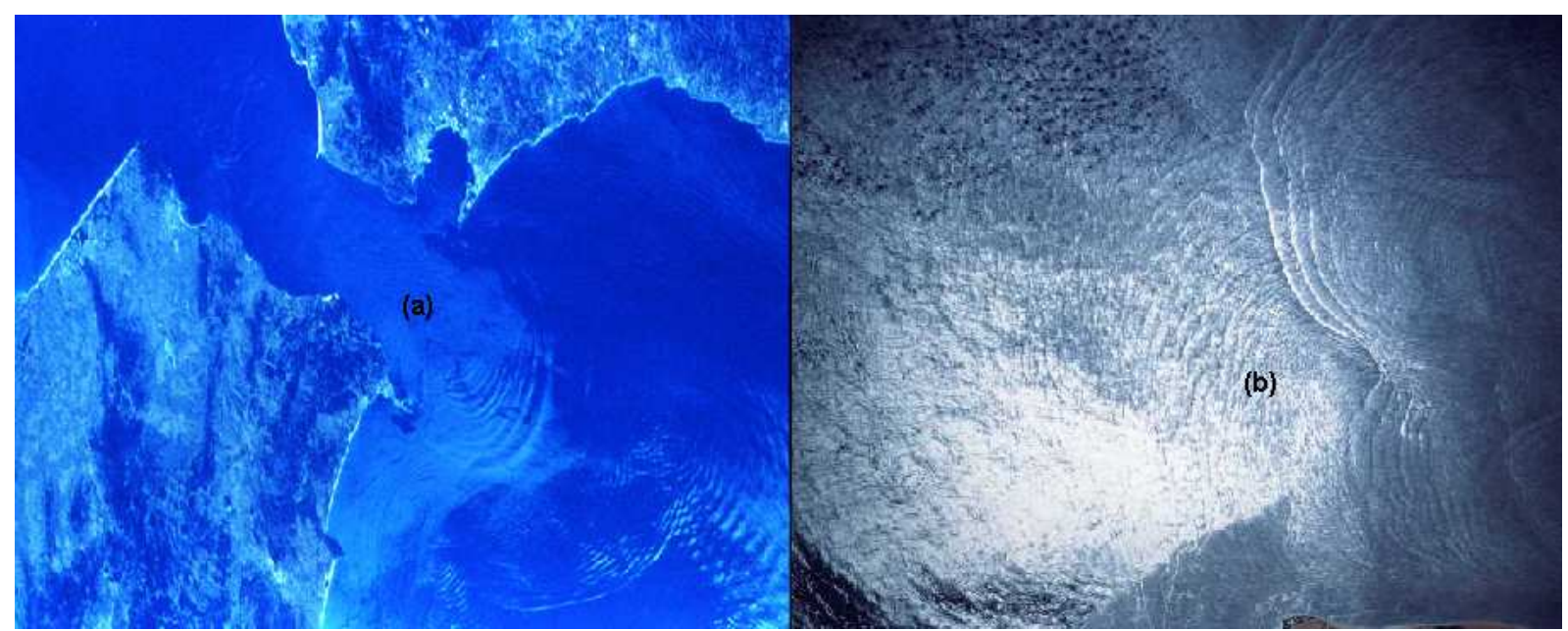

Figure 1: Solitons in the Gibraltar Strait (a) and solitons forming a Gaussian curve (b).

In 1834, a young scottish engineer, John Scott Russell (figure 2) was observing a channel boat when it suddenly stopped and a great mass of water arised and traveled by this channel apparently without change of form [5]. He was riding a horse and was capable of following the wave for one or two miles. He recreated the so called "Wave of Translation" in a experimental tank in his garden, but their results were ill-understood and ignored by contemporaries scientists because in the nineteenth century they were dealing only with linear differential equations which are not enough to model that phenomena. Linear waves can not propagate without change of form.

It was only in 1895, sixty one years after it was discovered, the "translation wave" began to be appreciated and explored by the KDV equation [6] and so on by Boussinesq and Rayleigh equations [7], etc. In 1965 Zabusky and Kruskall rebaptized the "translation wave" as "soliton" and with other scientists discovered several properties and conservation laws.

To form a soliton we have to get very special conditions. In one hand there is a dispersion effect (higher frequency components travel slower than lower ones) and in the other hand you have a amplitude effect (the top of the wave travels faster than the bottom part). We get a soliton when 


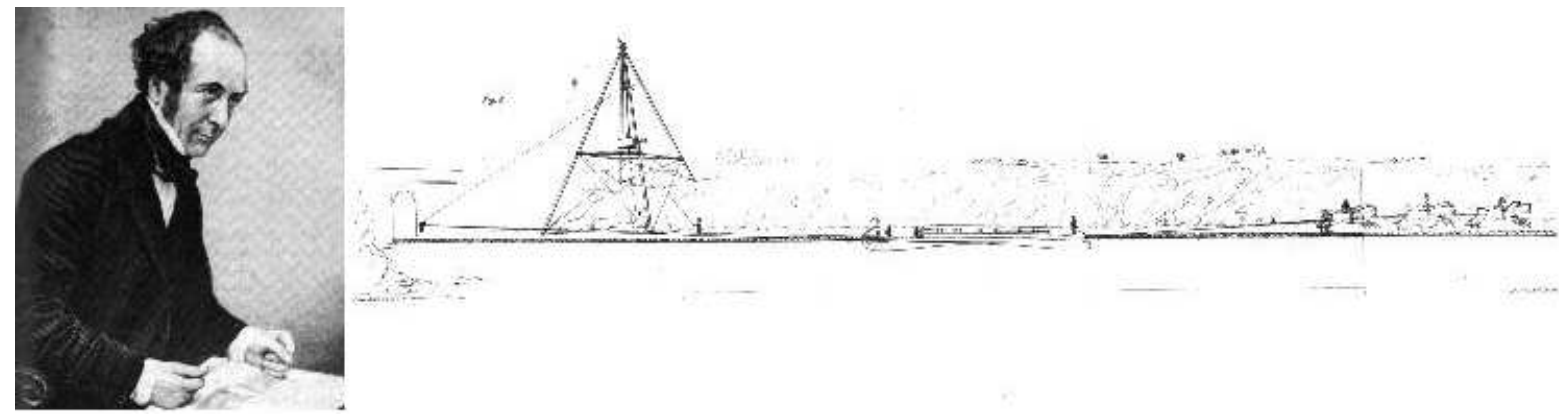

Figure 2: Russell and the wave of translation history.

those effects are in equilibrium. Which parameters in model equation lead us to a soliton solution ? That is the question we are interesting in.

The tidal bore, or in Brazil, the "Pororoca" (when the ocean water enters to a narrow river) is a example of how much special are the conditions to form a soliton. The Pororoca does not spread but it is not a soliton because the amplitude effect is not compensated, and one can see it is changing all long its passage. In other hand, solitons have very interesting properties, like in the KDV equation, where you can find one soliton solution which behavior is like two linear solutions respecting the linear superposition principle. You can find both, the Pororoca and a KDV two pulse soliton solution in figure 3 .

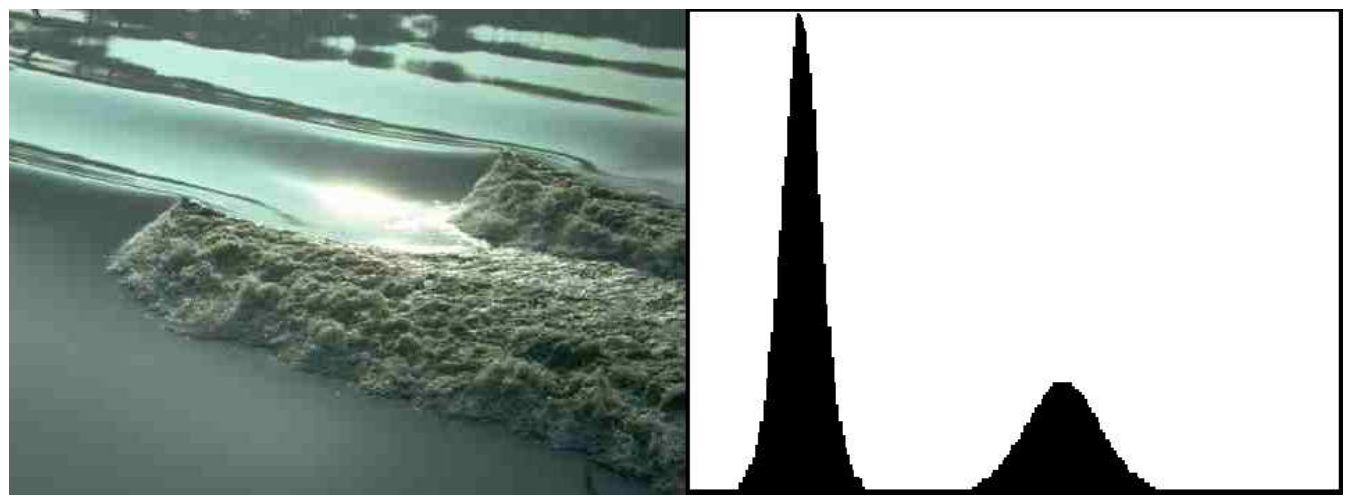

Figure 3: Pororoca in Amazonas river and a KDV two pulse soliton solution.

\section{Model Equation}

We investigate solitons in presence of surface tension related to reversible hamiltonian systems homoclinic solutions [8]. We say that a hamiltonian system is reversible if there is a involution $Q\left(Q^{-1}=Q\right)$ that is anticonical $\left(D Q^{t} \omega=-\omega D Q\right)$, and invariant by the Hamiltonian function $(H \circ Q=H)$. And we say that an orbit $\phi$ is homoclinic to a certain critical set $r$ of a dynamical system (it could be an equilibrium point or a periodic orbit) if the orbit is bi-asymptotic to this set, that is, $\lim _{t \rightarrow \pm \infty} \phi(t)=r$.

We use as a model equation a fourth order differential equation $u^{i v}+a u^{\prime \prime}-u+f(u, b)=0$, and the choice $f(u, b)=b u-\tanh (b u)$, which was based on [9],[10],[11],[12] and [13]. We can 
reformulate that model equation to a two degrees of freedom Hamiltonian system where $H=p_{u} v+$ $\frac{p_{v}^{2}}{2}+a \frac{v^{2}}{2}+\frac{u^{2}}{2}-\left(\frac{b u^{2}}{2}-\frac{1}{b} \ln (\cosh (b u))\right)$. The parameter $a$ is related to wave speed and parameter $b$ is related to surface tension.

We will work with two hypothesis. The system has non-empty unstable manifold and we have a reversibility function $Q$ for the system. In our model $Q\left(u, v, p_{u}, p_{v}\right)=\left(u,-v,-p_{u}, p_{v}\right)$ and its fixed points set is $\chi=\left\{\left(u, v, p_{u}, p_{v}\right) \mid v=p_{u}=0\right\}$. Our main theorem discussed in previous work [14] is : for Hamiltonian systems with a reversibility $Q$ and a equilibrium $r$, if $r \in \chi$ and the unstable manifold of $r$ intersects $\chi$, then there exists an homoclinic orbit to $r$. Based on this result we used a standart procedure: for a $(a, b)$ parameters grid we track the unstable orbit until it reaches the set $\chi$ of reversibility fixed points, determinig which values lead us to a homoclinic solution in the space $\left(u, v, p_{u}, p_{v}\right)$ and a soliton solution in coordinate $u$, that is, in our model equation. In figure 4 we found a cascade behavior (see [9] and [15]) for those values and, in figure 5, one can see soliton bifurcations for fixed $b=3$ and $b=4$, when parameter $a$ changes inside plotted regions of figure 4 $(i)$ and $(i i)$, provindig a geometrical point of view for phase and amplitude shifts phenomena.

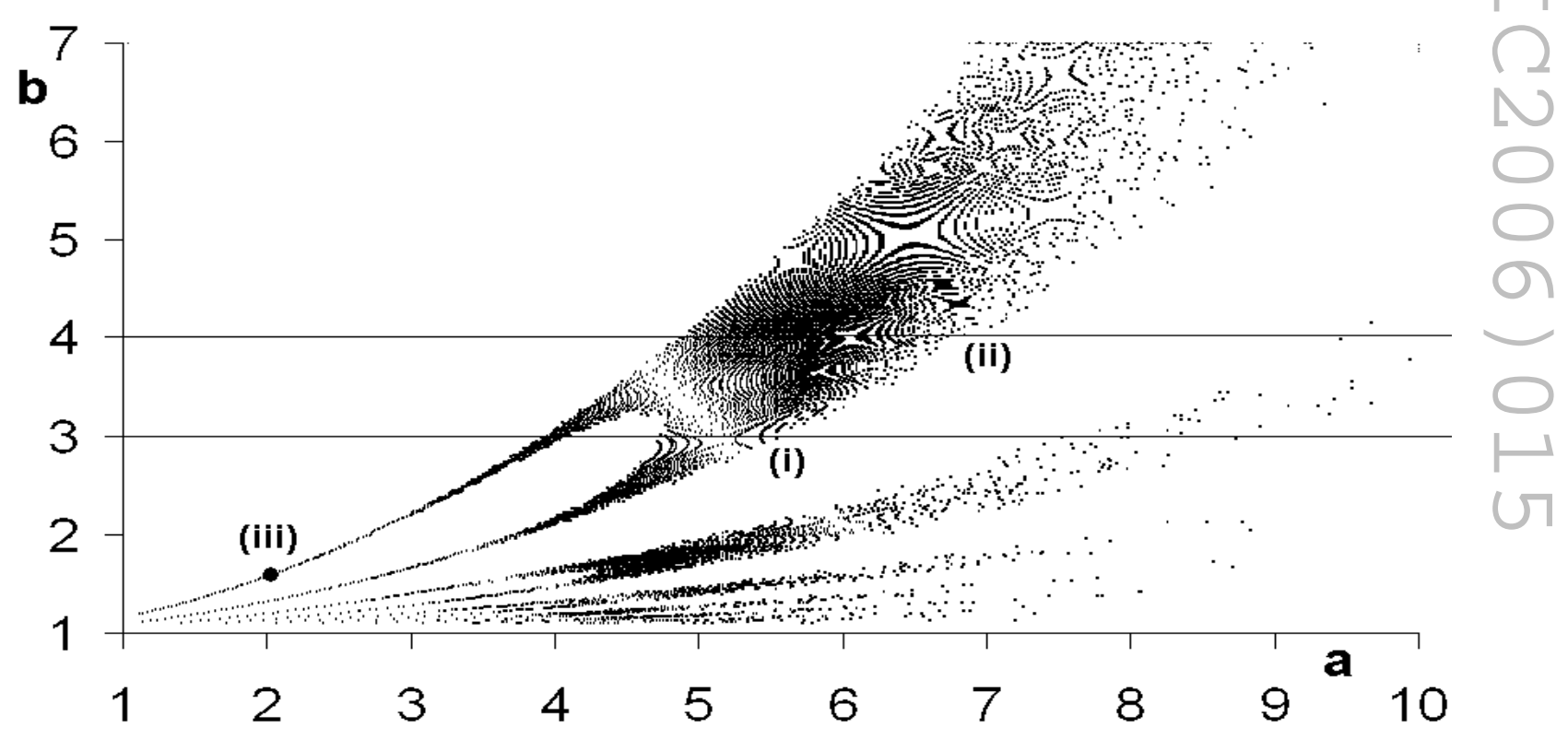

Figure 4: Soliton parameters.

\section{Energy surfaces and Poincaré sections}

Analysing our model equation energy and geometry, let $\Omega=\left\{\left(u, v, p_{u}, p_{v}\right) \mid H \equiv 0\right\}$ the zero energy surface, $\Sigma_{1}=\left\{\left(u, v, p_{u}, p_{v}\right) \mid p_{u} \equiv 0\right\}$ and $\Sigma_{2}=\left\{\left(u, v, p_{u}, p_{v}\right) \mid v \equiv 0\right\}$, the Poincaré sections related to the set $\chi$.

From the Hamitonian function we derive: $\Omega=\left\{\left(u, v, p_{u}, p_{v}\right) \mid p_{u} v+a \frac{v^{2}}{2}=-\frac{p_{v}^{2}}{2}-\frac{u^{2}}{2}+\left(\frac{b u^{2}}{2}-\frac{1}{b} \ln (\cosh (b u))\right)\right\}$.

In figure 6 one can observe a homoclinic bifurcation, lying in a projection of set $\Omega$, for a perturbation of order $10^{-2}$ to the parameters $(a, b)=(2.02,1.59)$, which were obtained from the algorithm (see the plotted point (iii) in figure 4). For those parameters, when the unstable orbit 

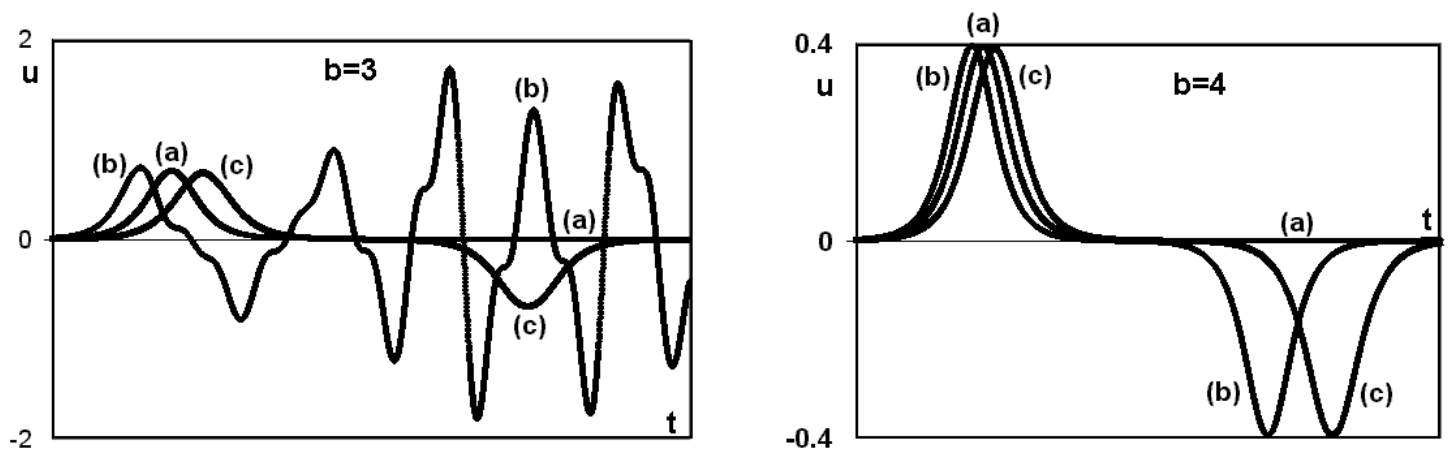

Figure 5: Soliton bifurcations.

instersects the set $\chi$ (rerversibility fixed points), represented by the line intersection between $\Sigma_{1}$ and $\Sigma_{2}$, we see some kind of "reflection", which connects the unstable and stable manifolds, forming a homoclinic orbit to the system orign (a) and, consequently, a soliton in model equation, as predicted by the main result. After parameters pertubation (b), we no long have that intersection, leading us to a oscillatory solution in the model equation.

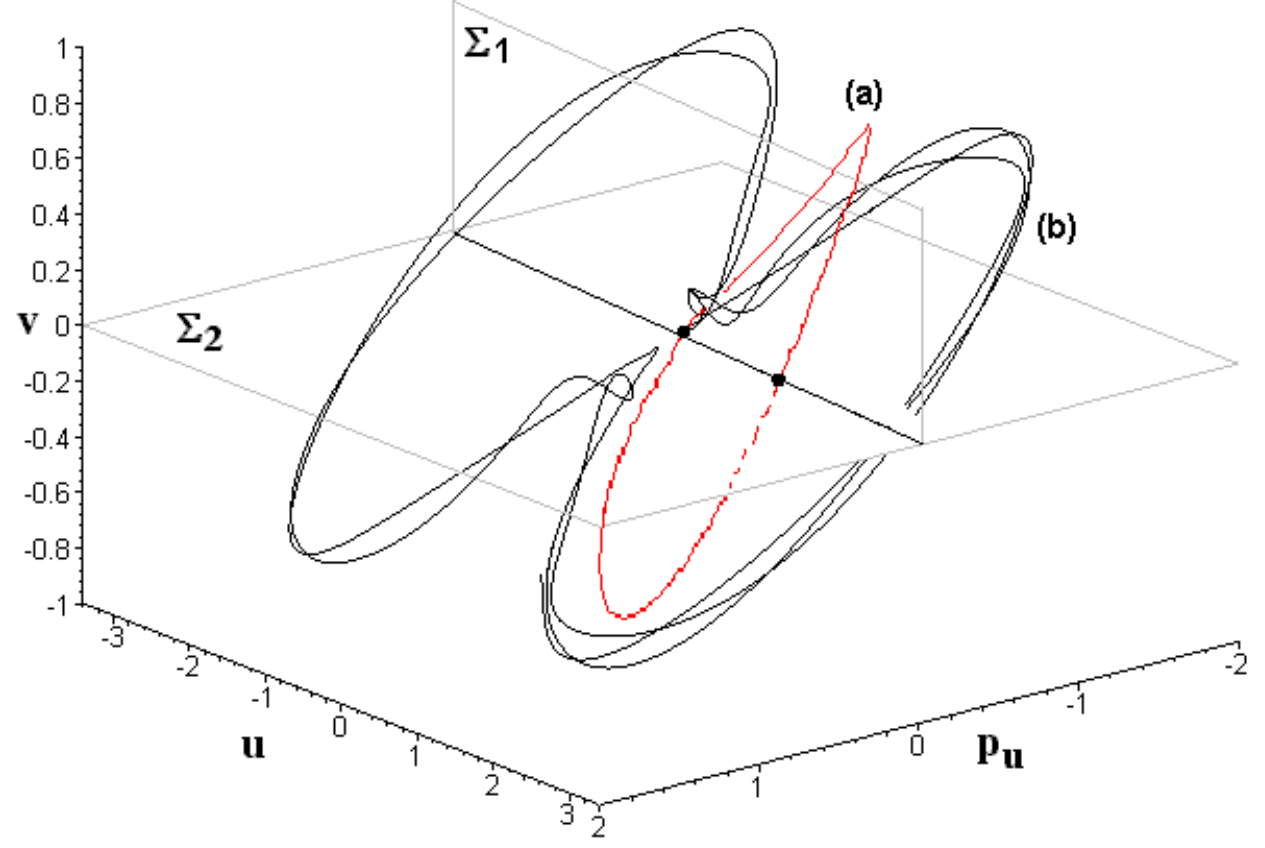

Figure 6: Homoclinic orbit (a) and bifurcation (b) for $(a, b)=(2.02,1.59)$ in model equation.

\section{Conclusion}

As pointed out in Section 2, the reversibility function plays important role in a pratical reformulation of usual methods for the situation where there is no simmetry associated to the Hamil- 
tonian system or it is unknown. Our approach is non restrictive and it is capable of providing a picture of geometrical structure for the usual solitons amplitude and phase shift phenomena. The cascade behavior obtained is consistent with experimental results. In Section 3 we can verify some geometrical aspects associated to energy surface and Poincaré sections involved in the main result. The search algorithm is simple (see [14] for more details) and can be generalized to a wide variety of systems by doing the necessary analytical and numerical work considerations, respecting the two hypothesis mentioned in Section 2. We are now investigation the possible fractal nature of figure 4 and we intend to report in more details the consequences of the method here presented.

\section{References}

[1] Cambridge University Press. Fluid mechanics. http://www.fluidmech.net/msc/f_linkw.htm, last access: october 2006.

[2] L. Zhang et al. Thermal properties of high temperature superconductors:. soliton statistics approach. Modern Physics Letters B, 11:149-154, 1997.

[3] Serge F. Mingaleev and Yuri B. Gaididei. Solitons in anharmonic chains with ultra-long-range interatomic interactions. Phys. Rev. E, 61:1044-1047, 2000.

[4] J. Brown. The secrets of everlasting life. New Scientist, pages 36-40, 1995.

[5] Department of Mathematics of Heriot-Watt University. John scott russell and the solitary wave. http://www.ma.hw.ac.uk/ chris/scott_russell.html, last access: october 2006.

[6] D. J. Korteweg; F. de Vries. On the change of form of long waves advancing in a rectangular canal, and on a new type of long stationary waves. Philos. Mag., 39:422-443, 1895.

[7] G. L. Jr. Lamb. Elements of Soliton Theory. Wiley, New York, 1980.

[8] C. J. Amick and K. Kirchgässner. A theory of solitary water waves in presence of surface tension. Arch. Rat. Mech. Anal., 105:1-49, 1989.

[9] A.R. Champnneys. Homoclinic orbits inreversible systems and their applications in mechanics, fluids and optics. Physica D, 112:158-186, 1998.

[10] C. G. Ragazzo. Irregular dynamics and homoclinic orbits to hamiltonian saddle-centers. Comm. Pure Appl. Math., 50:105-147, 1997.

[11] C. G. Ragazzo. Homoclinic orbits for $u^{i v}+a u^{\prime \prime}-u+f(u, b)=0$. Equadiff95: International Conference on Differential Equations, Singapore, World Scientific, 1998.

[12] B. Buffoni; A. R. Champneys; J. F. Toland. Bifurcation and coalescence of plethora of homoclinic orbits for a hamiltonian system. J. of Dyn. and Diff. Eq., 8:221-279, 1996.

[13] J. F. Toland and C. J. Amick. Homoclinic orbits in the dynamic phase-space analogy of an elastic strut. Euro. Jnl. Appl. Math, 3:97-114, 1992.

[14] Gerson Francisco and André Fonseca. Homoclinic orbits in reversible hamiltonian systems. Applied Mathematics and Computation, 176:654-661, 2006.

[15] A. Mielke; P. Holmes; O. O'Reilly. Cascade of homoclinic orbits to, and chaos near, a hamiltonian saddle-center. J. Dyn. Diff. Eqns., 4:95-126, 1992. 